Professor C C de Silva Oration - 2013

\title{
Paediatric Cardiology in Sri Lanka: Yesterday, today and tomorrow
}

\author{
Duminda Samarasinghe ${ }^{1}$ \\ Sri Lanka Journal of Child Health, 2014; 43(1): 3-19
}

(Key words: Paediatric Cardiology; Sri Lanka)

Cholmondely Chalmers de Silva, born on 25th February 1904, had his early education at St. Thomas' College, Mount Lavinia and entered Ceylon Medical College in 1922. After spending a couple of years in the medical college he joined University College London and Kings College Hospital and graduated MRCS LRCP in 1928. He left again to the United Kingdom in 1929, acquired MRCP London and returned to Ceylon, as Sri Lanka was then known, in 1931. In 1938 he returned to London to study for the MD and a year later he was back in Ceylon. In 1949 he joined the Faculty of Medicine, University of Ceylon as the first Professor of Paediatrics.

Professor CC's main clinical interests were nutritional disorders, haemoglobinopathies and infective diarrhoeas. Together with Dr. L.O. Abeyratne, he was instrumental in setting up the convalescent home at Thalagolla for nutritional rehabilitation of malnourished children. Professor $\mathrm{CC}$ was also a pioneer in the field of family planning contributing extensively towards it from about the mid-sixties. He was a wide-angled clinician and a great researcher. He held many posts in numerous professional organisations, both nationally and internationally. Pioneering work done by Professor $\mathrm{CC}$ would have definitely contributed to the very low infant and childhood mortality figures that we have achieved today.

When I read about Professor CC de Silva, I could not find a single sentence related to paediatric cardiology. But, ladies and gentlemen, unless Professor CC de Silva, as the first Professor of Paediatrics, and generations of his students that followed him, thought about the major killers at that time like malnutrition and diarrhoeal illnesses and brought them down to record low levels in the region, I would not be able to stand here to speak to you about paediatric cardiology today. Therefore, I consider Professor $\mathrm{CC}$ as a person who was instrumental in laying a firm foundation to what we are building today. I consider this as an honour and privilege to be able to deliver this year's Professor $\mathrm{CC}$ de Silva oration, and I thank the President and

$\overline{{ }^{1} \text { Consultant Paediatric Cardiologist, La dy }}$
Ridgeway Hospital for Children, Colombo, Sri
Lanka
Lanka the Council of the Sri Lanka College of Paediatricians for giving me this opportunity.

\section{Preamble}

Paediatric Cardiology is one of the most rapidly advancing fields in Sri Lanka. This advancement is not only in depth where it has advanced from simple to more complex lesions but also in breadth where it has advanced in the percentage of patients it could treat from a minority to a majority. However, we still have waiting lists and patients dying of cardiac conditions while awaiting surgery. Can we care for all children with heart disease in Sri Lanka, totally free of charge, at the cost of one billion rupees a year? This is the million dollar question that needs to be answered. As a developing country do we have the manpower and money? Can we be more cost-effective and is there any room for improvement from what we are doing at present?

The world has changed over decades and we are in the $21^{\text {st }}$ century. Fifty years ago, Toyota Motor Corporation changed from the conventional inventory system of purchasing to 'Just in Time' (JIT) manufacturing to cut down on inventory management cost and carrying cost. With these reforms, Toyota has become the leading motor manufacturing company in the world. Today, budget airlines provide the same basic service as conventional airlines at a fraction of the cost without compromising on passenger safety. With proper training and equipment, McLaren and Red Bull pit stop teams were able to change four tyres of a Formula One car in less than 2.5 seconds whereas it takes more than an hour at a tyre shop. By introduction of a production line concept, production time of Boeing 737 had dropped to 11 days while maintaining the highest quality and achieving the lowest $\operatorname{cost}^{1}$. When cardiac surgery costs 30,000 US dollars (USD) in USA and 5000 USD in private hospitals in India, Dr. Deve Shetty opens a new hospital in India targetting to provide cardiac surgery at $800 \mathrm{USD}^{2}$. In a world where people constantly change routines to do things faster, better and at a lesser cost, is there anything that we can learn from them to improve our existing system of Paediatric Cardiology? If we do so, we would be able to reach a point where we 
could provide care for all children with heart disease in Sri Lanka in the most appropriate and timely manner within the framework of a free health system of the country.

\section{What is Paediatric Cardiology?}

Paediatric Cardiology deals with all congenital and acquired heart diseases in childhood. It is a subspeciality which has great diversity and crosses a number of traditional medical boundaries. It covers all congenital heart lesions from fetus to adult. In addition, the work range of a Paediatric Cardiologist in our setup spans from clinical diagnosis to advanced echocardiography and from medical management of patients to therapeutic catheter interventions.

\section{History of Paediatric Cardiology}

The first pathologic description of a heart defect was made in 1513 by Leonardo da Vinci, who wrote: "I have found from $A$, left auricle, to $B$, right auricle, the perforating channel from $A$ to $B$ ", where he described a defect in the inter-atrial septum, known as Atrial Septal Defect (ASD) today $^{3}$. Paediatric Cardiology was limited to descriptive studies of congenital cardiac defects until Robert Gross ligated a Patent Ductus Arteriosus (PDA) in 1938 when Paediatric Cardiology, as a discipline, was born ${ }^{4}$. This highlights the importance of surgical association in development of the field. By 1961, Paediatric Cardiology became the first Sub-speciality Board in Paediatrics in the United States ${ }^{5}$. The past 60 years has brought enormous progress to the field. Cardiac Ultrasound, Colour-flow Doppler and Magnetic Resonance Imaging (MRI) have made diagnostic cardiac catheterization almost unnecessary. Instead, interventional cardiac catheterization has rapidly developed, has replaced surgery in the treatment of a number of cardiac defects, and has become complementary in many other conditions.

\section{Development of Paediatric Cardiology services in Sri Lanka: The past}

Development of Paediatric Cardiology in Sri Lanka did not happen overnight. In 1972, the Cardiology Unit at the National Hospital was commissioned and was the sole referral centre for adult and paediatric cardiac patients. Establishment of the Cardiology Unit at Lady Ridgeway Hospital (LRH) in 1999 with the appointment of a permanent Consultant Cardiologist, Dr. S. Narenthiran, was a major breakthrough in development of care for children with heart disease in Sri Lanka. The unit had only a small room with an echocardiography machine, a medical officer, a nurse and an attendant. A 24 bedded ward came up in 2002 to facilitate in-ward treatment. Commissioning of the Cardiac Catheterisation Laboratory in December 2005, which paved the way for diagnostic and therapeutic cardiac catheterisations of children, was a giant leap forward. With this, the main components needed to provide Paediatric Cardiology services at LRH were instituted.

However, Paediatric Cardiology could not stand alone without Cardio-thoracic Surgery and Intensive Care. Before 2007, all children with heart disease needing surgery were referred to Sri Jayawardenapura General Hospital, National Hospital or Karapitiya Teaching Hospital. These hospitals were already overburdened with adult patients awaiting coronary artery surgery. Therefore, children with more complex congenital heart lesions with more demanding surgery and poorer outcome were obviously not the surgeon's first priority. The affluent went abroad seeking treatment. Some others, to save their beloved child sold everything they had, including the house they lived in, to seek treatment offshore or in the private sector. To address this long standing issue, the Cardio-thoracic Unit, two Cardiac Operating Theatres and a 6-bedded Cardiac Intensive Care Unit (ICU) were declared open in January 2007. Before long, it was felt that a 6-bedded ICU was not enough to handle the output of two operating theatres. To fill the gap, a 12-bedded High Dependency Unit (HDU) was built and commissioned in 2010. As this too will not be adequate to handle the patient load, further expansion with a four storied twin building was planned. With this expansion, infrastructure facilities to handle children with cardiac problems in Sri Lanka will be adequate. However, provision of infrastructure does not ensure that it will cater to the patients in the best possible way. Manpower, a ready supply of consumables and optimal utilization of available resources are mandatory to provide the best possible outcome.

\section{Introduction of Paediatric Cardiology as a sub- speciality in paediatrics}

When should a country introduce a government sponsored Paediatric Cardiology Programme is always a question especially in developing countries. It is a fact that Congenital Heart Disease (CHD) is a major killer in children and a main contributory factor to infant mortality. However, in a country where there are children dying of simple diarrhoeal diseases and respiratory tract infections, which can be controlled at a much lower cost, should we embark on a government sponsored Paediatric Cardiology Programme? 
Different countries introduced Paediatric Cardiology as a separate speciality at different times. In USA it was in 1961, in Cuba it was in 1986 and in India it was in 1990s. The best example of a scientific way of introducing a government sponsored Paediatric Cardiac Programme comes from Cuba. They observed that Infant Mortality Rate (IMR) had dropped to below 20 (19.6 per 1000 live births) by 1980 from 38.7 in $1970^{6}$. As infant mortality declined CHD became increasingly predominant as a primary cause of death in infants less than 1 year of age. Analysing the issue they have strengthened their Paediatric Cardiology Programme and as a result the IMR in Cuba has steadily declined to the current level of 5/1000 live births. In India, it was the opposite and Paediatric Cardiology Programme commenced in 1990s when the IMR was close to 80 . This was not a government sponsored programme but initiated by a few Paediatric Cardiologists and Cardiothoracic Surgeons who were trained abroad, returned to India and realised the lack of services available even to those who could afford care in the private sector. Therefore, in India, where IMR is still close to 50, there are many state of the art Paediatric Cardiac Centres in the private sector. In Sri Lanka, the introduction of Paediatric Cardiology Programme was due to a change in the paediatric curriculum in 2001. The two year training was extended to three years and there was discussion about introduction of sub-specialities in paediatrics. When the first batch of candidates of the new curriculum passed out in 2004, Paediatric Cardiology was offered as a sub-speciality. However this was about 5 years after bringing down the IMR to a value below 20 .

\section{The ground situation in Sri Lanka}

The problem that paediatricians faced was clearly highlighted in a study conducted in the Professorial Paediatric Unit at LRH in 1999 on children with CHD. According to that, within a period of one year, case fatality rate was $18 \%^{7}$ among such children and CHD was the second commonest cause of deaths in the unit. It further stated that unless surgical care for patients with CHD in the public sector was improved, many such children would continue to die early due to lack of facilities.

Analysing the disease burden is the most important step in planning. It is estimated that approximately 400,000 live births occur in Sri Lanka each year. As the incidence of CHD is 6-8 per 1000 live births $^{8}$, it can be estimated that the number of children born with CHD is 2427-3236 each year in Sri Lanka. According to the literature, two-third of this (1618-2157) will need surgery or intervention for their heart lesion. If properly treated, $85-95 \%$ of patients born with congenital cardiac malformations will reach adulthood ${ }^{9}$. With these estimates, our target should be to treat around 2000 children every year. However, when we consider the numbers awaiting surgery in waiting lists and those who will need re-operations in the future, we should plan for approximately 2500-3000 surgical procedures and catheter-based interventions to be performed every year.

\section{Resources available in Sri Lanka}

Lady Ridgeway Hospital for Children is the only tertiary care referral centre for children with heart disease in the country at present. There are three Paediatric Cardiologists, three Cardio-thoracic Surgeons and four Cardiac Anaesthesiologists at present. In addition, there are Paediatric Cardiologists at Kandy, Karapitiya, Kurunegala and Jaffna hospitals. In almost all major hospitals there are adult cardiologists who will do the initial assessment and refer the problematic patients for further opinion to a centre with a Paediatric Cardiologist. Even though there are Cardio-thoracic Units at National Hospital, Sri Jayewardenepura General Hospital (SJGH), Karapitiya Teaching Hospital and Kandy Teaching Hospital, since the inception of the Cardio-thoracic Unit at LRH, their focus has shifted towards coronary artery disorders and adult CHDs, leaving almost all children with CHD to be treated at LRH.

\section{Paediatric Cardiology services at LRH}

The Paediatric Cardiology Unit at LRH has 3 basic components: the Outpatient Clinic, the Cardiology Ward and the Catheterisation Laboratory. The first contact of almost all patients with suspected heart disease is the Outpatient Clinic. In more than 95\% of patients, the diagnosis and management plan is decided after the initial assessment. A minority of patients will need further evaluation with a cardiac catheterisation before a final diagnosis and plan of management is formulated.

\section{Out-patient Clinics}

On the average, we see 10,000-12,000 new referrals every year (Table 1 ). In addition, we have our follow up patients which amounts to another 12,000-15,000 per year (Table 1). As we do an echocardiographic assessment in almost all patients, we perform approximately 25,000 echocardiograms each year. There is no waiting list for assessment as we do not give appointments for first visits. From September 2009 onwards, all clinic visits including echocardiogram reports are entered into a database specifically designed for the purpose. 
Table 1: Clinic attendance by year from 2007 to 2012

\begin{tabular}{|l|c|c|c|c|c|c|}
\hline \multicolumn{1}{|c|}{ Year } & $\mathbf{2 0 0 7}$ & $\mathbf{2 0 0 8}$ & $\mathbf{2 0 0 9}$ & $\mathbf{2 0 1 0}$ & $\mathbf{2 0 1 1}$ & $\mathbf{2 0 1 2}$ \\
\hline No. of clinics & 313 & 285 & 315 & 335 & 314 & 316 \\
\hline No. of first visits & 11,424 & 11,271 & 10,465 & 10,119 & 10,474 & 9,875 \\
\hline No. of revisits & 12,914 & 13,108 & 12,267 & 13,695 & 17,743 & 15,123 \\
\hline Total patients & 24,338 & 24,379 & 22,732 & 23,814 & 28,217 & 24,998 \\
\hline Average/clinic & 78 & 86 & 72 & 71 & 90 & 79 \\
\hline
\end{tabular}

Clinic attendance at LRH varies from 40-200 patients a day whereas it is $10-20$ patients in the West. Does this mean that we do a substandard evaluation? Our system is different. We work like the team in a Formula One Pit Stop where there are different categories of people assigned for specific jobs. In the West it is like a wayside tyre shop where every job is done by a single person. So a tyre change which takes 30 minutes at a wayside tyre shop is done in 2-4 seconds in a formula one Pit Stop. In our system, the cardiologist mainly focuses on a quick examination and the echocardiogram where there is a nurse to register the patient and keep him or her quiet during assessment, a medical officer to read the referral, enter the data and write a back referral, if needed, another medical officer to explain the condition to the parents and a nurse to tell them about the next visit and follow-up plan. In addition, there are attendants to line up the patients for echocardiograms so that there is no delay. Therefore, we do not have a waiting list to assess first referrals. In the West the waiting time could range from 4-6 months. Should we also follow the same Western setup and keep the patients in suspense for months to years before giving them a date for evaluation or triage the patients and spend more time with the more complex ones and less time with normal patients is a question that needs to be answered.

In Sri Lanka, we have a free referral system. As we do not have guidelines or restrictions for referrals, even Grade I soft systolic murmurs are sent for cardiac evaluation. According to the data available in our clinic database, analysis of first referrals by age shows that more than $33 \% \quad(n=13,097)$ of patients were referred within the first year of life and $58 \%(n=23,125)$ were referred before 5years. This increases our work load and limits the time that is available to spend on assessment of more complex cases. However, the favourable side of it is that we diagnose most of the conditions very early in life. We rarely get a Transposition of the Great Arteries (TGA) diagnosed later than one month in life or a shunt lesion which is missed in childhood and has become inoperable. Prior to the introduction of the clinic database, we did a study analysing 5,000 first referrals made from July 2008 to January 2009 to the Cardiology Unit at LRH (Figure 1). This showed that most of the referrals made in infancy had a positive diagnosis compared to a minority being positive when referred after 5 years of age.

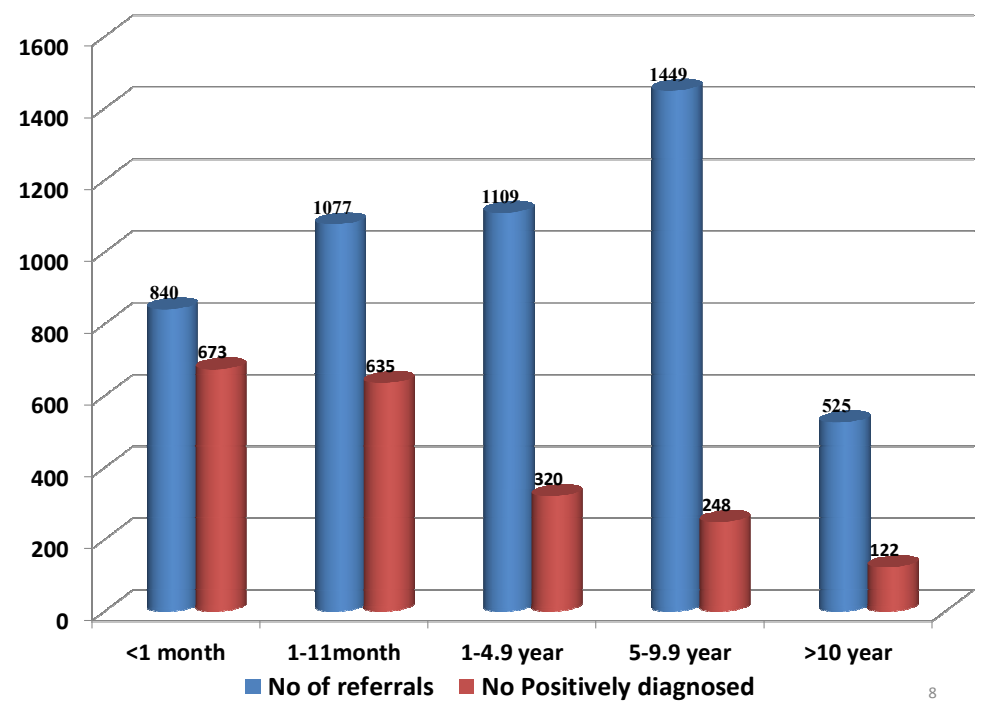

Figure 1: No. referred \& diagnosed to have a cardiac lesion by age (n=5000, cardiac lesion-1998 (40\%)) 
The most common reason for referral was a murmur but only $40 \%$ of such referrals had a positive diagnosis (Figure 2). Out of all reasons for referral, cyanosis had the highest positive predictive value which was $90 \%$ (Figure 2). Unfortunately we do not check for desaturation in all newborn babies even when a pulse oximeter is available in the ward. It is not included in the neonatal examination check list and if included will help to diagnose most of the cyanotic heart diseases at birth.

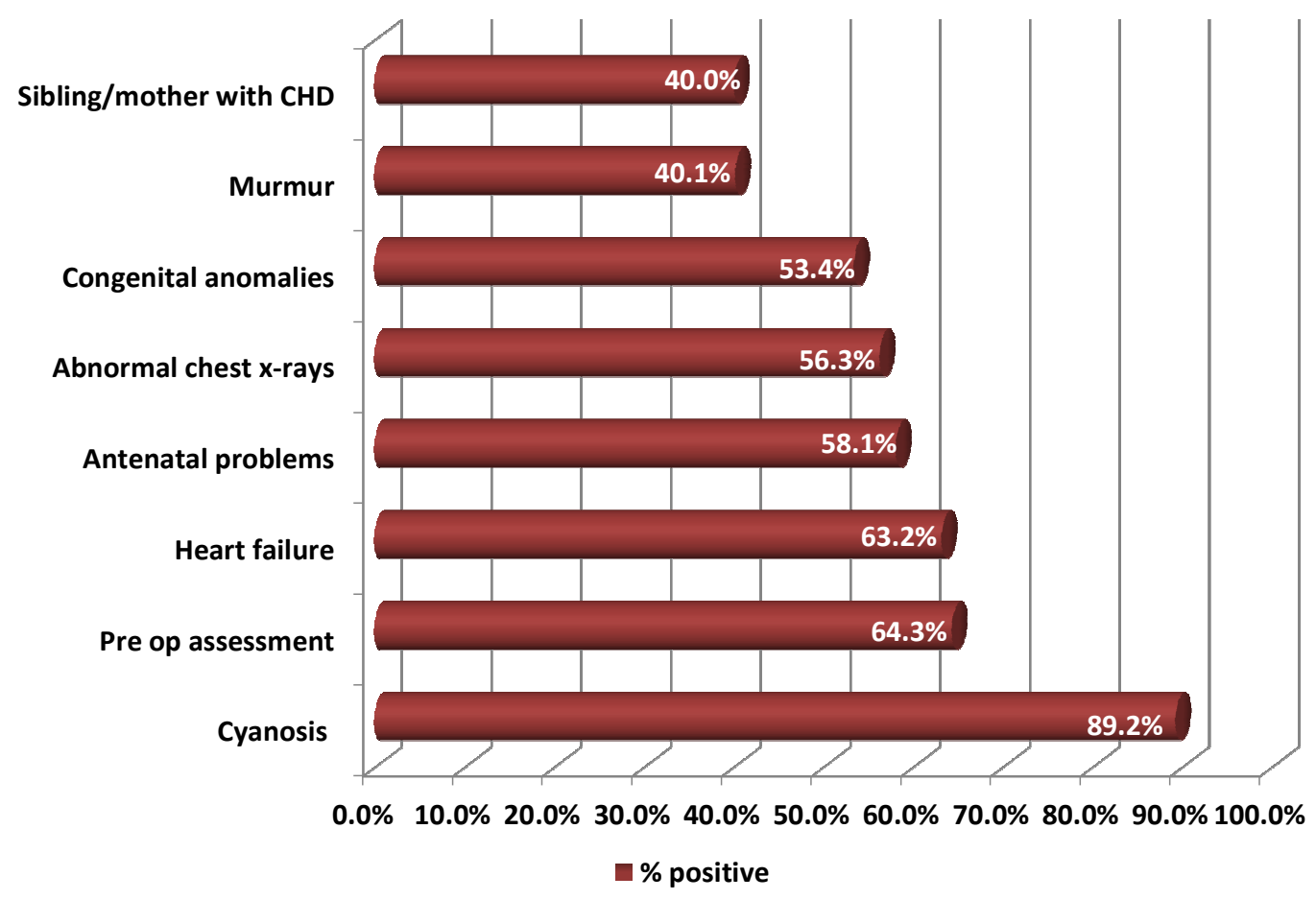

11

Figure 2: Reasons for referrals with highest positive predictive value

\section{Cardiology Ward}

Bed occupancy rate of the 24-bedded Cardiology Ward was more than $90 \%$ over the past 5 years. Analysis of 2012 admissions $(n=1871)$ in the ward database showed that $56.8 \%$ were admitted for cardiac catheterisation and $29.3 \%$ were for surgery. During 2012, the average number of days spent in the hospital before being taken up for surgery was 9.85 when admitted for surgery. Average total number of days spent in hospital when admitted for a catheterisation was only 2.61 . This clearly shows that the current surgical output is inadequate to handle the patient load.

\section{Cardiac Catheterisation Laboratory}

Cardiac Catheterisation Laboratory at LRH was the first in Sri Lanka which could be opened for emergencies 24 hours a day, 365 days a year and is the only dedicated Paediatric Cardiac Catheterisation Laboratory in the country. Waiting time for an elective catheter intervention is around 2-3 months and is progressively coming down. However, depending on the urgency, procedures can be done even on the same day. In our catheterisation database, we have analysed the districts of residence in patients who have undergone catheterisations from January 2012 to June 2013 (Figure 3). This shows that a large number of patients were from Colombo $(n=183)$, Gampaha $(\mathrm{n}=167)$, Kurunegala $(\mathrm{n}=135)$ and Kalutara $(n=103)$ districts and very few patients were from Vavuniya ( $n=9)$, Mullaitivu $(n=6)$, Mannar $(n=5)$ and Kilinochchi $(n=2)$ districts. However, when the percentage of patients from each district is compared to the percentage of population in each district, it clearly shows that this discrepancy is purely because of the population density in each district. This proves that the Catheterisation Laboratory at LRH provides a national service even though it is located in Colombo. 


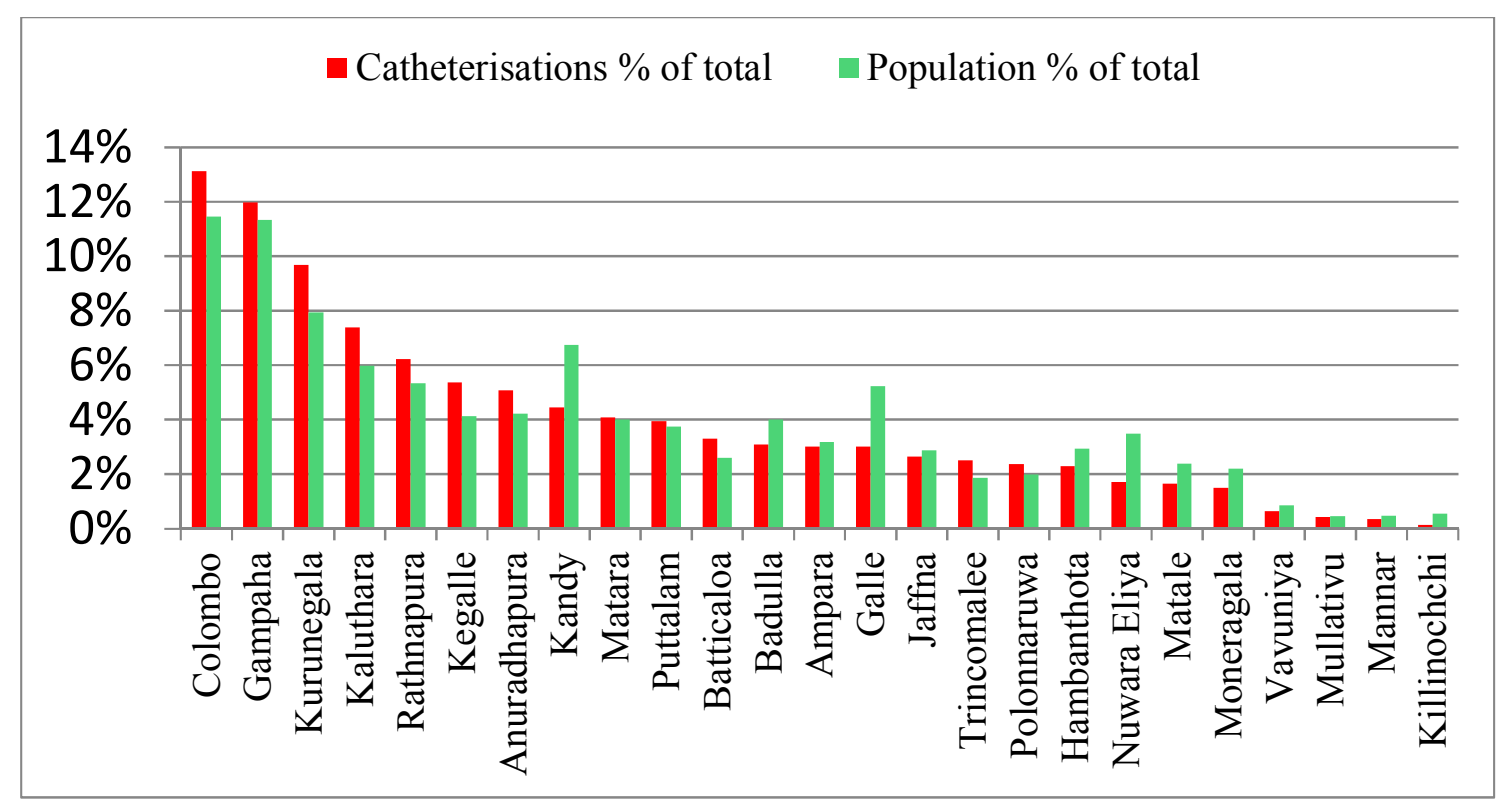

Figure 3: Percentage of catheterisations by district of residence from January 2012-June 2013 (n=1403) against percentage of population in each district

From December 2005 to June 2013, 6300 cardiac catheterisations were performed and the mean weight of the patients was $14.4 \mathrm{~kg}$ (range $1.9 \mathrm{~kg}$ to $67 \mathrm{~kg}$ ) and mean age was 4.49 years (range 1 day to 54 years). Even though LRH is a dedicated children's hospital, $8 \%$ of cases performed were more than 12 years of age. Out of 6300 catheterisations, $33 \% \quad(n=2101)$ were diagnostic cardiac catheterisations and $66 \%(n=4140)$ were interventional procedures (Table 2). In addition there were 7 hybrid interventions which were performed together by a Cardiologist and a Cardiac Surgeon and 52 radiological procedures which were performed by a Radiologist. Further analysis of radiological procedures was not done as they were non-cardiac procedures. All procedures were done under x-ray guidance. Fluoroscopy time provides an indication of the duration of the procedure and radiation exposure. Mean fluoroscopy time was slightly higher in diagnostic catheterisations (11.7mins) when compared to that of interventional procedures (8.74mins).

Table 2: Type of cardiac catheterisations performed in catheterisation laboratory from December 2005 to June 2013

\begin{tabular}{|l|c|c|c|}
\hline Type of procedure & Number & \% of total & Mean fluoroscopy time (minutes) \\
\hline Interventional & 4140 & $65.71 \%$ & 8.74 \\
\hline Diagnostic & 2101 & $33.35 \%$ & 11.7 \\
\hline Radiology & 52 & $0.83 \%$ & 17.8 \\
\hline Hybrid intervention & 7 & $0.11 \%$ & 7.04 \\
\hline Total & 6300 & $100.00 \%$ & \\
\hline
\end{tabular}

The number of cardiac catheterisations has gradually increased from 661 in 2006 to 1024 procedures in 2011 and has reached a plateau of around 1000 cases since then. Common interventions included device closure of ASDs and PDAs, Pulmonary and Aortic Balloon Valvuloplasty, Balloon Angioplasty of Coarctation of Aorta and palliative procedures like Balloon Atrial Septostomy. Some other rare and exotic interventions include perforation and opening of atretic pulmonary valve in newborns with Pulmonary Atresia and intact ventricular septum, closure of Coronary Arterio-venous (AV) Fistula, closure of Pulmonary AV Fistula, closure of systemic to pulmonary fistula, device closure of Main Pulmonary Artery (MPA) after bidirectional Glenn shunt and device closure of Aortopulmonary Window.

\section{Device closure of ASD}

Device closure of ASD was the commonest catheter intervention performed in our Catheterisation Laboratory. From December 2005 to June 2013, a total of 1716 patients were taken up for device closure of ASD. $85 \%$ of the patients 
were between 4-12 years of age and $65 \%$ were female. Devices from 5 different manufacturers; Amplatzer, Blockaid, Cardiofix, Cocoon and Lifetec were used with similar results. The procedure was successful in 1612 (94\%), unsuccessful in $95(5.5 \%)$ and abandoned in 9 $(0.5 \%)$. Success rate of ASD closure has significantly improved from $75 \%$ during the initial 3 years to $97 \%$ during the last 3years (Chi square $37.1, \mathrm{df}=1, \mathrm{p}<0.001)$. Failures were more in females $(7 \%)$ compared to males $(3 \%)$ (Chi square $9.2, \mathrm{df}=1, \mathrm{p}=0.002)$. This was probably because even with suboptimal margins, females were taken up to attempt device closure due to the fear of the scar that they would get with surgery. Mean fluoroscopy time has significantly dropped from 16.2 minutes in 2006 to 4.1 minutes in 2013. Device embolisation occurred in $8(0.46 \%)$ and all were within 24 hours of implantation. One patient died due to cardiac perforation and $4(0.23 \%)$ patients developed complete heart block after device closure. There were no cases of endocarditis or bacteraemia after the procedure. Compared to statistics from the initial period, our latest statistics show less complications and very high (almost $100 \%)$ procedure success rate. This is partly due to improvement in procedure skills and more stringent case selection criteria for device closure.

\section{Device closure of PDA}

This was the second most commonly performed procedure at our Catheterisation Laboratory. From December 2005 to June 2013, 1631 patients were taken up for PDA closure. Success rate was 91\% $(n=1488)$. In $3 \%(n=43)$ the procedure had failed and in $6 \%(\mathrm{n}=100)$ closure was not attempted. A single coil was used in $763(51.3 \%)$, multiple coils in $129(8.7 \%)$ and a device in $596(40 \%)$ patients. Median weight was $9.3 \mathrm{~kg}$ (range 3 to $58 \mathrm{~kg}$ ) and $173(10.6 \%)$ patients were less than $6 \mathrm{~kg}$. Success rate was $86.3 \%$ during the initial 3 years and $98.2 \%$ during the last 3 years (Chi square 48.0, $\mathrm{df}=1$, $\mathrm{p}<0.005$ ). Mean fluoroscopy time was 17.01 minutes in 2006 and has dropped to 4.72 minutes in 2012. Embolisation of coil or device occurred in 28 $(1.7 \%)$ patients. There were no deaths, bacteraemia or endocarditis following PDA closure, indicating that it is a safe procedure.

\section{Rare interventions}

From December 2005 to June 2013, 6 patients had undergone device closure of Aorto-pulmonary Window, 9 had undergone closure of Coronary Artery Fistulae and 18 patients were taken up for perforation of atretic pulmonary valve. These were some of the rare and more technically challenging procedures that we have performed in the Catheterisation Laboratory. In addition, we have performed 7 hybrid procedures during this period with the help of the Cardio-thoracic Surgeons.

\section{Complications in cardiac catheterisation}

Complications in cardiac catheterisation are very rare. They can range from a simple haematoma at puncture site to death on the table due to cardiac perforation. We have analysed our major complication rate from December 2005 to June 2013. Total number of patients was 6300. Our major complication rate, where there was either loss of life, significant life threatening event or permanent disability as a complication of the procedure, was $1.36 \%$ in 2006 and has remained less than $0.3 \%$ from 2009 to 2013 . There were 17 deaths $(0.27 \%)$ during the study period but only 3 $(0.05 \%)$ were a direct consequence of the procedure. There was a statistically significant difference in mortality rate between initial 2 years $(0.76 \%)$ and last two years $(0.05 \%)$ (Chi square $7.9, \mathrm{df}=1, \mathrm{p}=0.004)$. During the study period, 67 patients had significant complications needing prolonged hospital stay or an additional intervention. Minor complications were prospectively recorded during the last 16 months and the rate was $5.15 \% \quad(n=68)$. The two commonest minor complications were weak lower limb pulse due to femoral artery puncture and fever which consisted of $75 \%$ of total minor complications. There were no cases of bacteraemia or endocarditis following cardiac catheterisation during the whole study period.

\section{Have we lowered the pole? Comparable data from other studies}

Airline industry is one of the most highly regulated and provides the safest mode of transport in the world. The target of Boeing 737 manufacturing line is to do it at the fastest and the lowest cost with highest quality, every time the task is performed. As health care providers, shouldn't it be our target as well? In Sri Lanka we cover a large number of cases in a short period of time with minimal resources and at a very low cost. By doing so have we lowered our standards is the question. In device closure of ASDs, the mean fluoroscopy time was 16.2 minutes in 2006 but has dropped to 4.1 minutes in 2013. The mean fluoroscopy time ranged from 17 to 20.3 minutes in other published studies $^{10,11}$. This shows that we have become faster with time. One main reason for this shorter time is that we have omitted balloon sizing of the defect. By becoming faster have we compromised on safety? Embolization of ASD device after deployment is one of the main complications seen in device closure of ASD. Incidence of device embolization is about $0.55 \%$ ( 1 in 200 cases) in experienced hands ${ }^{12}$. Our overall device 
embolization incidence was $0.46 \%$ and we have not had a single device embolization during our last 500 cases. This indicates that by becoming faster, we have not compromised on patient safety.

Unlike surgical closure of ASD where the procedural attempt success rate is almost $100 \%$, in device closures there is a chance of failure. The procedural success rate in ASD device closures is around $93-98 \%$ in most of the reported studies ${ }^{11,13}$. Our success rate was 75\% during 2006 and has improved to $97 \%$ in 2012 and $100 \%$ in 2013. This indicates that by becoming faster we have not compromised on our success rate.

When we consider our overall data, the major complication rate was $0.83 \%$ in $2006-2007$ and has dropped to $0.21 \%$ during the period from January 2012 to June 2013. Incidence of major complications in diagnostic or therapeutic cardiac catheterisations ranges from $0.14 \%$ to $11 \%$ according to various studies ${ }^{14-16}$. This indicates that our safety standards are comparable to most of the published data and by performing the procedures with minimal resources we have not compromised on patient safety.

\section{Are we working in isolation?}

Team approach in comprehensive cardiac care In today's context, paediatric cardiac care is delivered by the united effort of Paediatric Cardiology, Paediatric Cardio-thoracic Surgery, Paediatric Cardiac Anaesthesia and Intensive Care Teams. There are many other ancillary services like Infection Control, Radiology and Physiotherapy which are equally important for the best possible outcome of the patient. This is called Comprehensive Paediatric Cardiac Care ${ }^{17}$.

\section{Evolution of team approach}

Over the years, Paediatric Cardiology centres have changed from the linear model to a team approach Earlier model for management of CHD was linear, where the cardiologist makes the diagnosis, refers the patient with the diagnosis to the surgeon and the surgeon operated based on the written records. The patient was sent to the ICU after surgery and managed based on the operation note written by the surgeon. This was practical for simple lesions like ASDs, PDAs and simple ventricular septal defects (VSDs). But when surgical techniques improved and more complex patients were operated upon, this linear model failed. Instead, centres which adopted the team approach provided the best outcomes. In the team approach the decision is made collectively by the Cardiologists, Surgeons and Anaesthesiologists. If and when indicated, opinion is taken from other specialists like Paediatricians, General Surgeons, Neurologists, Radiologists, Nephrologists and Clinical Geneticists. Patients who are waitlisted for surgery and those who need critical decisions are discussed at a combined meeting where all parties present their views. With advancement in knowledge about heart disease and with availability of a multitude of management options, collective decision making has provided immunity, at least partly, against litigation as well. Therefore, in the current context Comprehensive Paediatric Cardiac Care through team approach has become the main decision making process in the management of children with heart disease.

\section{Cardiac Surgery}

Cardio-thoracic Surgery at LRH commenced, in 2007 January with two Operation Theatres, two Surgeons and one Anaesthetist. There were only 6 ICU beds which was the main limiting factor in the number of surgical procedures that could be performed. To address this issue plans were laid for a 12-bedded HDU which was completed in 2010. In addition there was a new Surgeon and an Anaesthetist appointed in 2009. With this, the number of surgical procedures per year has gone up from 273 in 2007 to 882 cases in 2012. 


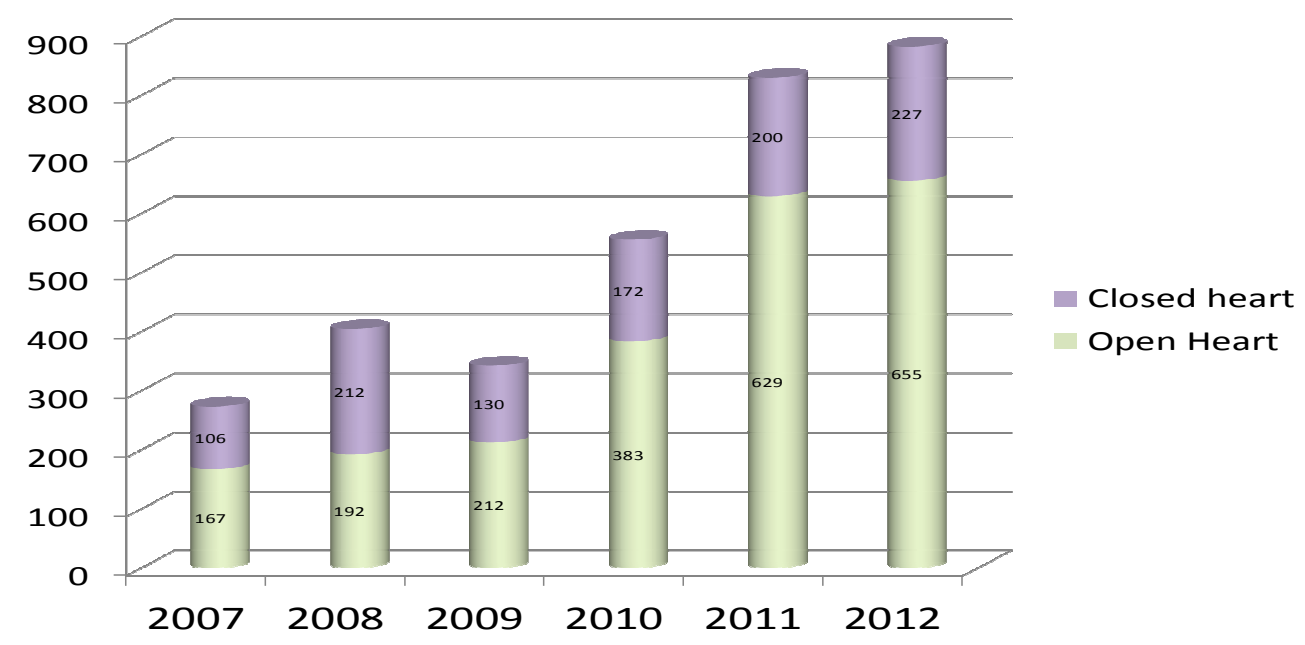

Figure 4: Paediatric cardiac surgeries at LRH from 2007 to 2012

Success of a programme is not measured by the number of surgical procedures alone. It involves case mix, age and weight of patients operated and mortality rates as well. We have analysed the surgical procedures performed from January 2010 to June 2012. We have performed procedures ranging from simple ASDs to more complex cases like TGA and univentricular repairs. The mortality rate from January 2012 to May $2013(n=1300)$ was analysed and it showed that the overall mortality rate was $6.38 \%$ with $6.48 \%$ for open heart surgical procedures and $6.12 \%$ for closed heart surgical procedures, which is comparable to Western figures ${ }^{18,19}$. Another indicator of success of a Paediatric Cardiac Surgical Programme is the weight of the patient at the time of surgery. Today at LRH a clear majority of operations are done on children less than one year of age with a weight less than $10 \mathrm{~kg}$ and all newborn surgical procedures like correction of TGA and Total Anomalous Pulmonary Venous Drainage (TAPVD) are done during the neonatal period. This breaks the earlier myth which postulated that cardiac surgery can be performed only when the child becomes $10 \mathrm{~kg}$.

\section{Paediatric Cardiac Anaesthesia and Intensive Care}

These are an integral part of care in paediatric heart disease. We started the ICU with 6 beds in 2007 and expanded it to 18 beds by 2010 . The plan is to expand it to 24-30 beds within the next 2-3 years with completion of the new building. ICU beds are mainly used for post-operative patients. Generally ICU beds are the main limiting factor in most of the Paediatric Cardiac Programmes but with the expansion we will be able to eliminate that factor in our hospital.

\section{Future of paediatric cardiac services in Sri Lanka}

If we are to plan the future of Paediatric Cardiology we need to adopt what Paul McCartney said; "Think globally, act locally". Being a developing country we cannot afford to do all the procedures that a centre in a developed country does. But, at the same time we cannot neglect our children with heart disease just because we are a developing country. Therefore, we need to forecast, set our goals, prioritize patients and move forward in the most cost-effective way. If we plan our strategy well, we will be able to provide care for most of the children with heart disease in Sri Lanka, even with the limited resources available.

\section{Future goal in Paediatric Cardiology}

There is little doubt as to what the goal should be as invariably it should be to provide care for all children with heart disease in the most appropriate, timely and cost-effective manner. Based on the available statistics, we should plan for around 2000 cardiac surgical procedures and 1000 catheter based interventions each year to reach that goal. In Australia, where the population is equal to that of Sri Lanka, 1900 cardiac surgical procedures are performed every year to provide a good quality service ${ }^{20}$. Therefore our estimate is a realistic figure. The question is whether we have the manpower and funds to reach this goal.

\section{Is this a reachable target?}

When compared to the targets of some of our neighbouring countries, ours is a reachable target. In India there are 180,000 born with CHD and they 
provide surgery to only 6500 a year while in China there are 132,000 children born with CHD and only 60,000 are operated ${ }^{20}$. Compared to these figures we need to increase our numbers by only 1000 cases per year to care for all children with heart disease which is a reachable target.

\section{Cost of cardiac care}

\section{Cost and cost-effectiveness of cardiac catheter interventions}

Catheter interventions are costly due to the high cost of consumables and the cost of infrastructure development. A Catheterisation Laboratory costs about Rs. 80 million and installation and building costs another Rs. 40 million. A Catheterisation Laboratory so established lasts only about 10 years. In addition, the service agreement costs approximately Rs. 8 million a year. Therefore, we have to spend approximately Rs. 20 million a year for the machine alone. In such a situation, if we perform 1000 cases a year, cost per case for the machine alone is Rs. 20,000. If we perform only 500 cases per year this cost will rise to Rs. 40,000 per case and will not be cost-effective as the cost of a diagnostic cardiac catheterisation in a private hospital including consumables, staff salaries and stay is about Rs. 35,000. We have calculated the cost of consumables used for procedures performed in 2010 at LRH and the approximate cost was about Rs.100 million. Some of the catheter interventions like device closure of ASD can cost up to Rs. 350,000 per patient and most of the patients will not be able to afford such high costs.

In a country like Sri Lanka, whether these interventions are cost-effective is the question that needs to be answered. Different countries have different cut off levels for cost-effectiveness of medical interventions. It is expressed as the cost per Quality Adjusted Life Year (QALY) that the patient gets after the intervention. In USA it is 50,000 USD per QALY and in the UK it is 20,000 $\mathrm{GBP}^{21}$. The World Health Organisation gives a cut off for developing countries and according to that, an intervention which costs less than the per capita GDP of that country per a QALY is very costeffective $^{22}$. According to World Bank statistics, the per capita GDP of Sri Lanka is 2923 USD. As almost all our catheter interventions provide many decades of normal life to that child, all our interventions fall under the very cost-effective category.

\section{Who spends for the procedure?}

According to World Bank publication 'Good Practices in Health Financing', in low income countries most of the health expenditure comes as private out of pocket expenditure ${ }^{23}$. Contribution from the governments and private pooled, i.e. insurance schemes, is low. This changes when the country develops into a high income country where most of the expenditure is borne by the government and insurance companies and only a minimum amount comes as private out of pocket expenditure. According to Sri Lankan statistics in 2005, even though well known for its free health service, private sector expenditure was $53 \%$, out of which $49 \%$ was household out of pocket expenditure ${ }^{24}$.

The question is whether this is the same for cardiac interventions in Sri Lanka. To assess this we have analysed the device closure of ASDs done in our unit. The device costs 175,000 SLR and due to lack of devices supplied through the government we issue letters to the President's Fund for financial assistance. President's Fund pays approximately 150,000 SLR for the device. The balance is paid by the parents or is taken from the Social Services Department if the parents cannot afford it. The analysis of 1155 cases done at LRH showed that more than $99 \%$ of the time the device was either supplied by the hospital or the President's Fund. All other procedures done at the Catheterisation Laboratory were done totally free of charge and therefore provided entirely by the government. This is true for Cardiac Surgery and Anaesthesia as well. This indicates that even though overall health statistics show that $49 \%$ is out of pocket expenditure in Sri Lanka, when it comes to high cost procedures like catheter interventions and cardiac surgery, more than $99 \%$ are done at government hospitals free of charge.

When a significant number of adults with coronary artery diseases go to the private sector for treatment, why do children with CHD come to the government sector? In my view this is mainly because CHDs are diagnosed early in life and the parents are young and have just started to earn. They have spent most of their savings on the wedding, antenatal care and the delivery. If a cardiac lesion is diagnosed in the newborn, they do not have any financial reserves to spend on surgery or intervention in the private sector. Moreover, congenital heart lesions are not covered under insurance. Therefore, if we are to provide care for children with heart disease in a country like ours, the only option is to develop the government sector services. Introduction of a fee levying system for those who could pay and introduction of an insurance scheme which covers CHD are other options to at least partly cover the expenditure.

\section{Cost forecast}

Cost can be divided into cost of 2000 cardiac surgical procedures and 1000 catheter interventions. The cost of consumables used for a single open heart surgical procedure is approximately Rs. 145,000 . In addition, there are 
other costs like salaries of staff, water, electricity, drugs, equipment and maintenance, which are important in running a programme. When all this is considered, it will cost approximately Rs. 400,000 per cardiac surgery in the government sector whereas it costs around Rs. 600,000 in the private sector. Therefore, to perform 2000 cardiac surgical procedures per year we should allocate Rs. 800 million a year. Similarly cost of 1000 catheter based interventions will be approximately Rs. 200 million. In summary, we need to spend approximately one billion rupees a year to treat children with heart disease in Sri Lanka.

In a country which has a health budget of 70 billion rupees a year, can we ask for 1 billion rupees to treat children with heart disease alone? Can we provide free of charge treatment to all children with heart disease? What do other countries do? Can we make it more cost-effective and sustainable? Is the private sector an alternative? Should we charge the patients in the government sector? These are some of the key issues that need to be addressed.

\section{How can we reach this target?}

\section{Catheter based interventions}

At the moment at LRH we do approximately 1000 cardiac catheterisations every year and out of that more than $60 \%$ are therapeutic interventions. Our waiting list for a catheter intervention has been about 2-3 months for the last 2 years and urgent cases can be accommodated even on the same day. Therefore, having one dedicated Paediatric Catheterisation Laboratory is more than enough to cater to the needs of the Sri Lankan population. However, to keep the hands-on-training of the other Paediatric Cardiologists, either they can come to LRH or can share the outstation Catheterisation Laboratories with the adult cardiac team. There are 4 Catheterisation Laboratories outside Colombo viz. Kandy, Karapitiya, Kurunegala and Jaffna. In three of them Paediatric Cardiologists share the Catheter Laboratory with adult cardiologists. One day to paediatrics is generally the rule in most such systems in other countries as well. Therefore, if they do 5 cases a week in each Catheter Laboratory that will amount to 1000 more cases a year at peripheral Catheter Laboratories. With the 700 cases done at LRH it will be nearly twice the required number of interventions for Sri Lanka. This shows that in catheter based interventions, we already have reached the target and have infrastructure and manpower even to serve double that targetted number.

\section{Cardiac surgery}

Starting with 273 cases in 2007, the number of cardiac surgical procedures done at LRH has gradually increased to approximately 900 cases per year in 2011 and 2012. The realistic target should be 2000 surgical procedures per year to provide optimal care. How are we going to bridge the gap? The simple answer would be to divide the balance and hand them over to the other four Cardiothoracic Units in the country which are mainly handling adult coronary artery disease. If they are also overworked, the solution will be to build another one or two similar Paediatric Cardiothoracic Centres for the country. However, when scientifically analysed, it is evident that these two options are perhaps not the best solution to the problem. It is an established fact that a majority of heart diseases should be operated within the first year of life and a significant number will need surgery within the first 3 months. It is also known that centres which operate only on children provide a better outcome than centres which operate on both children and adults. Moreover, it is a proven fact that when complex CHD is considered, centres which do larger volumes of surgery provide better outcome when compared to centres which do smaller volumes ${ }^{19}$. Worldwide, governments are realising that maintaining a few high volume centres is more cost-effective than maintaining many low volume centres. Therefore, in the UK, four low volume centres were closed bringing down the total number of Paediatric Cardiothoracic Centres from 11 to 7 and the ultimate target was to have only 6 such centres for the whole of the $\mathrm{UK}^{25}$. When manpower is considered, we do not have adequate Cardio-thoracic Surgeons to manage even the existing Cardio-thoracic Units. Therefore, building another unit is not a practical solution. Furthermore, one needs to undergo Cardiac Surgery once in a lifetime and there are very few emergencies in Paediatric Cardiac Surgery. Any patient can be transported by road maximally within 6-8 hours even from the furthest point in the country. Even if we airlift those 2-3 patients who need urgent cardiac surgery every year, it will be cost-effective in the long run. Therefore, if we are to provide good quality service to all patients with CHD in Sri Lanka, the best option is to strengthen the cardio-thoracic facilities at LRH to reach the target of 2000 surgeries a year, rather than planning to open a number of smaller centres around the country.

\section{Reaching the targets in the most cost-effective way}

\section{Do we have the best system in the world?}

The first step in changing a system is scientific analysis of the existing system to see whether it is efficient, cost-effective and provides good quality care in a timely and appropriate manner. In healthcare delivery, we still follow the same system which was planned many decades back when there was no air transport and all items ordered took 6 
months to reach from the West, there were no mobile phones and all urgent correspondence had to be made via telegrams, there were no computers and all data had to be meticulously recorded in books and there were no sub-specialities and provision of free health care was restricted to 4 major specialities. Are there ways of managing the existing system in a better way and what are the deficiencies in the current system? We will analyse a few issues we have noted that could be improved for better productivity.

\section{Few thoughts for improvement}

\section{Use the existing infrastructure to the maximum of its capacity}

If there is a waiting list for catheter interventions, whether it is cheaper to pay the staff for extra hours of work and use the existing Catheterisation Laboratory after working hours or establish a new Catheterisation Laboratory to handle the extra cases is the question. Establishing a Catheterisation Laboratory is a costly affair. Most of these costly equipment last only about 10 years maximum as the technology gets outdated and manufacturers do not provide parts after about 10 years. Therefore, we need to use this equipment maximally within the 10 years. In Sri Lanka, all Catheterisation Laboratories in the government sector work only from $8 \mathrm{am}-4 \mathrm{pm}$ and are closed for 16 hours a day. If we are to build a new Catheterisation Laboratory to accommodate the balance 300 catheter interventions (to reach our target of 1000 cases/year from the current 700 cases/year) we need to spend Rs.20 million a year only for the infrastructure (as shown in previous section on cost and cost effectiveness of catheter interventions). But, if we are to pay the staff Rs.20,000 per case and use the existing Catheterisation Laboratory after normal working hours to cover the additional 300 cases, we need to spend only Rs. 6 million every year. With this, the government can save Rs. 14 million a year and even the Rs. 6 million that we pay to the staff will stay inside the country. The same principle can be applied for Cardiothoracic Surgery or any other field where the initial establishment cost is extremely high.

\section{Purchasing consumables; purchasing in bulk vs just in time manufacturing}

According to the procurement process in Sri Lanka, all consumables, except those that are urgently required, need to be requested through the Medical Supplies Division and are purchased in bulk. We order our consumables annually and during this process we need to predict the items needed for the coming year. It is a difficult task as we cannot predict the sizes of ASD devices that will be used in the coming year as it all depends on the sizes of the actual defects in individual patients. Therefore, at the end we are left with sizes which have not moved fast. This also happens to surgical items like valves and conduits which get expired after some time. Moreover, some of the orders are processed many years after placing the order and by the time the items are supplied, the procedure is abandoned due to complications associated with it. One good example is the supply of 40 perimembranous devices in 2010 which were ordered in 2006. The procedure had been abandoned by that time because of associated complete heart block in children. Sometimes orders placed over many years are supplied at once. Thanks to this we now have the largest collection of PDA coils in the world. Furthermore, if there is a quality failure it takes a long process to return the items and on certain occasions it is impossible to return. In addition, we need storage facilities to store the stocks and some get destroyed due to poor storage. When it comes to payments, we pay for the items months to years ahead of the actual date of use. In summary, we use a procurement system which was initiated decades back and was given up by leading companies all over the world due to issues associated with it.

'Just in Time' (JIT) manufacturing is the system adopted by many world renowned companies today to purchase consumables. This was introduced by Toyota Motor Corporation in 1950s as Toyota Production System (TPS) and later changed and adopted by many other companies. The principle is that suppliers supply the items directly into the production line or few days before, minimising the need for storage. World's largest aeroplane manufacturer, Boeing, manages their supply chains with such efficiency that most of the parts come directly into the production line from thousands of suppliers around the world. They have changed their systems as they have understood over time, purchasing in bulk is not the most cost-effective way. We at LRH, practise the same principle on a smaller scale when purchasing ASD devices when they are not available through the government sector. The supplier directly supplies the device on the day of the procedure and if it is used for the patient we issue a letter to the President's Fund and the President's Fund makes the payment to the supplier. It is the responsibility of the supplier to stock all sizes and provide good quality devices. If there is a quality issue we return it immediately and use another device. With this, we do not have the hassle of storage and stock maintenance. If JIT manufacturing is proven to be better and is adopted by many world renowned companies, why cannot we adopt the same principle when purchasing consumables through the government?

\section{Purchasing equipment; quality vs. cost}

One other major issue we face when ordering equipment through tender procedures is getting 
good quality equipment. Echocardiography machine is one example. Echocardiography is the main diagnostic tool in heart disease in children and is the decision making tool. Outcome in echocardiography depends on three factors: the operator, the machine and the time available for evaluation. Each can compensate for the other to some extent. As the operator is static and cannot be changed, if we need to do more number of assessments in a given time, we need a good machine which can provide good quality images. Even though purchased at a higher initial cost it will pay back in the long run. Cheaper machines with poor quality assessment will pay back in lives in the long run. If we purchase a low quality machine and make errors in diagnosis because of the poor image quality, nobody will accuse the machine for the errors made. Therefore, when purchasing equipment we have never compromised on quality and have done whatever possible to maintain the standards. In my opinion the tender procedure, even though thought to be fool proof, paves the way to dump low quality products that cannot be used to deliver a quality service at a relatively high price. It is similar to purchasing a car through tender procedures and getting a Morris Minor instead of a Mercedes Benz because the cost quoted for the Morris Minor is slightly lower than that for the Mercedes.

\section{Minimization of in-hospital days}

One of the main aspects conveniently forgotten in our government hospital setup is the cost of keeping patients in-ward. We do between 4-10 cardiac catheterisations per day, 5 days a week and all those patients are admitted the day before the procedure and are discharged on the following day. All patients are given dates using the clinic database and if there is any need to postpone, they are informed beforehand. This way, we minimise the number of in-patient days and manage the ward with only 27 beds.

When planning hospitals in Sri Lanka, we plan the hospital to keep patients in hospital for procedures but not to perform more procedures and send the patients home. Therefore, most of the hospitals have large numbers of beds instead of more Operating Theatres, Catheterisation Laboratories, Computer Tomography (CT) or MRI scanners. The Royal Children's Hospital in Melbourne which provides a similar in-patient service has only 334 beds but at LRH we have about 1000 beds. Therefore, if we are to provide a cost-effective service we need to improve facilities to do more procedures and investigations and not facilities to keep more patients in-ward for such procedures.

\section{Cost-effective staff management}

Cost-effective management of staff is a very low priority issue in the government service. Administrative rules and Establishment Code overrules cost-effectiveness, common sense and efficiency. There is no staff appraisal or incentives for those who work harder. From its inception in 2005 we had an on-call staff for the Catheterisation Laboratory and the time to open the Catheterisation Laboratory in an emergency was agreed upon to be one hour. The nurses and technicians came from home and they were paid only if they came for a procedure. However, over time administrators changed and at one time a decision was made by the nursing administration that one hour was not acceptable according to the prevailing rules and nurses were ordered to be in-house during off hours. When all other staff including Radiographers, Consultant Anaesthetists, on-call Anaesthetist and Consultant Cardiologist came from home, what was the purpose of having a nurse in- house? Even though we have written a letter to the effect explaining the expenditure incurred to the government, the decision was not revised and continued for nearly one year and the government paid overtime to the two nurses who stayed overnight. During this whole period we did only 23 procedures and none of them needed immediate opening of the Catheterisation Laboratory. This is only one example where the existing laws need to be changed depending on the need to run a programme in the most cost-effective way.

\section{Manpower and training}

The two main issues with manpower resources in the future will be Paediatric Cardio-thoracic Surgeons and nurses. Even though Paediatric Cardio-thoracic Surgery was introduced as a separate sub-speciality about 4 years back, nobody has taken it up. This lack of interest in Paediatric Cardio-thoracic Surgery is not specific to Sri Lanka but is a worldwide issue. This is mainly because of the complexity of the job with less revenue for the time spent. To address the issue in the UK they have introduced better payment schemes for Paediatric Cardio-thoracic Surgeons. To perform 2000 surgeries a year we need a minimum of 5-6 surgeons at LRH. Unless we plan for it now, we are going to face a significant manpower crisis in the near future.

The same issue applies to nursing staff as well. At the moment the Cardio-thoracic ICU is managed with only $60 \%$ of the nursing requirement. There is significant mal-distribution of nurses in the country as they always prefer to serve in their home towns due to the high cost of living in Colombo. However, under government salary scales they are not entitled to a better salary just because they work in a highly demanding Cardio-thoracic ICU 
in Colombo. The only solution to this as well as to the long waiting list is introduction of after-hours surgery at LRH through the President's Fund or donor agencies. But, even at the moment, cardiac theatres function till late in the evening on most of the days. So they are already working after hours for no extra payment at present. Introduction of after-hours surgery will therefore provide some incentive for them to stay at LRH.

In sub-specialities in medicine, selection is purely on knowledge but not on skills specifically required for that speciality. In contrast, selection test for an Air Traffic Controller is mainly skills based where skills needed to handle air traffic like multi-tasking, numerical skills, IQ, memory and speech are tested in the selection examination. Then they are trained and only at the exit examination the theory knowledge is tested. Is this not important in certain fields of medicine as well? If we adopt the same policy in medicine we will be able to deliver a more efficient and better quality service for the same amount of money spent.

In a world where the processing power of the computer doubles every two years but that of the human mind remains the same, how do we cope with the advancement in medicine and technology as healthcare professionals? The only way to handle this is to narrow down the subject that each person handles and continue with the same for life as it takes about 10,000 hours of practice for one to become proficient in a field. But in our setup, which was introduced when there were no computers and medicine was at a primitive stage, we transfer all categories of staff except Consultants from one speciality to another every 45 years. In today's context, do we gain anything by doing this? There should be a system where other categories of staff should have the liberty to stay in a specific field if they achieve some skill in that field over time. Then only the fields will progress as Consultants alone cannot provide a good quality service.

\section{Paediatric cardiac services in Sri Lanka: What is the future?}

When building and supporting paediatric cardiac services in developing countries, different governments and organisations adopt different policies. The first and easiest way most charities do is to collect some patients from a developing country and take them to a developed country to do surgical procedures. Yet others organise a whole team of Cardiologist, Surgeons, Perfusionists and nurses to fly over to a developing country where there are some facilities to operate and do a few surgical procedures every year. Under both these schemes they generally do a maximum of 30-40 patients a year and there is no follow up for these patients in the long run. But both these schemes are very successful in raising funds, as they are very appealing and can collect huge sums of money. However, both these programmes will not deliver the best for the money spent, as only a few of the thousands affected will get some cure. It is an established fact that if a cardiac programme is to be successful, it has to be managed by the local team. The visiting teams should target strengthening the local team and should not be interested in the number of cases that they operate on a given visit. In Sri Lanka too, the first two schemes prevailed for a long time without much impact on the real issue. The issue got addressed, at least partly, with the introduction of the third system. Therefore, for the future as well, strengthening the local team is the best way to address the issue.

\section{Ways of strengthening local resources}

\section{Expansion of infrastructure}

To perform 2000 surgical procedures a year we need at least 4 Operating Theatres. With the proposed twin building for cardiac care at LRH we will be able to reach that target within the next 2-3 years. In addition, we will be expanding our ICU strength to 24-30 ICU beds. The Cardiology and Cardio-thoracic Wards will have more beds for pre and post-operative patients. With this new building coming up in 2-3 years, we will have the infrastructure needed to cater to almost all children with heart disease in Sri Lanka.

\section{Introduction of new technology and services}

A human tissue bank and extracorporeal oxygenation are the two new additions that we are planning to introduce in the near future. The tissue bank will provide homografts and other tissues necessary for repair of certain types of heart disease. Homografts are the best and most costeffective solution compared to the alternative artificial materials available at present. Nearly half of the funds necessary to establish the facility are already raised and the project will be completed within 1-2 years.

\section{Ready supply of consumables}

A ready supply of consumables is a major issue that we are going to face in the future. If we are to treat all children with CHD free of charge we should have a budget of one billion rupees a year. Re-sterilization and re-use of items, use of cheaper alternative products, low cost modifications of procedures to cut down on budgets (e.g. use of multiple coils for PDA closure in place of a costlier PDA device) are some of the alternatives that centres in this part of the world follow to keep their programmes sustainable. Introduction of insurance schemes, promotion of donor agencies or charities 
for support or introduction of a fee levying system (depending on the income of parents) at least to cover the cost of consumables, are some practical alternatives to a less sustainable "total free for all" system. These schemes together with the free health service will be able to provide a good quality service free to those who need a free service and at a reasonable cost to those who can afford.

\section{Efficient record keeping and clinical database management}

If we are to tell the world what we do in a scientific way we need to maintain proper records. The most efficient way of doing this is to have a clinical database. One might think that addition of a clinical database will increase the workload of staff and will hamper clinical work. If properly constructed, a clinical database will cut down the work of all categories of staff and they will have more time to attend to real clinical work in the ward. If one considers the time a medical officer spends on filling investigation request forms, writing referral letters, tracing investigation reports from a pile of investigations and writing discharge summaries, one can imagine the time saved by using a properly designed database. The same applies to nurses who register all patients when they get admitted to the ward (who are already registered at the admission counter), enter all accountable drugs and other material in different books, spend time on balancing drugs and write lengthy midnight reports leaving aside real clinical work. Attendants and other staff are no exception as they have to go to the laboratory many times a day to collect reports, go to the Radiology Department to get ultrasound scans (USS) or CT appointments and to the indoor dispensary and various other places to hand over the drug orders and balance sheets that nurses have spent so much time on. When they go to these places they do not use the staircase but use the elevator and incur a heavy electricity bill to the hospital as well. We should also consider the amount of paper wasted for this process and the amount of storage space needed to store this paper load for years and years. Even if meticulously done, there is no easy way of assessing and analysing these written records and therefore they cannot be used for any forecasting or other similar purpose. So if one critically and scientifically analyses the issue and constructs a database to address all these issues it will save a lot of manpower and resources to the government and from a fraction of the money thus saved, full infrastructure needed to establish such a database can be built

\section{Until we reach the maximum capacity}

At present we are able to provide surgical facilities to only about $50 \%$ of the patients who need surgical intervention. Even if we reach the full capacity in 3-4 years, until such time we need an interim plan to provide maximal benefit to society through the limited resources available.

\section{Prioritization of scarce medical resources}

Prioritisation of medical resources is a constant debate. Even though, we do not have a waiting list for catheter interventions, there are thousands of patients waiting for surgical intervention. In such a setup what policy do we have for prioritizing patients? Out of the commonly used principles of allocating scarce resources like lottery, first come first served, sickest first, youngest first, number of lives saved, prognosis and social usefulness, are perhaps some of the criteria that could be used for some sort of prioritization for surgery.

Even though we do not have a strict prioritisation policy, we do apply a combination of some of these policies when prioritizing patients. We always consider the sickest first along with the best outcome. For example, a VSD patient on a ventilator will be given priority over a hypoplastic left heart syndrome. Although we have a waiting list we cannot adopt the first come first served policy. It will make so many children like TGA, large VSDs and AV canal defects inoperable or succumb to their illness while patients who came first but who could wait longer like ASDs will be operated on. Lottery is obviously not a good option as there are many other variables that determine the outcome. Youngest first is also not applicable as all our patients fall under young category. In addition, we also consider the social usefulness to some extent as we give priority to those who do not have other co-morbidities. However, a hard and fast prioritization policy is not practical as all these methods of prioritization have their own flaws. Some other factors that need to be considered are the commitment of the family for further care, background environment and chances of the patient dying of another issue and the possible contribution of the patient to the society in the future.

In Cuba, which had a very good Paediatric Cardiology Programme, the heart centres had to cut back on the number of cases during an economic crisis in the early $1990 \mathrm{~s}^{6}$. Those patients who could wait were waitlisted and those who would die if not operated on, were given priority. Less costly offpump surgical procedures were given preference over more costly pump procedures. During this period, till 1997, the number of surgical procedures was halved compared to the previous years. Since 1997, conditions have steadily improved and now again there is no waiting list for Paediatric Cardiac Surgery in Cuba. We also can apply the same principles and make the best use of the resources currently available to provide surgery to those who really need and leave the patients who can wait to 
be operated once the system becomes better in a few years. However this needs meticulous planning, organisation and commitment of the staff as well as parents.

In conclusion, Paediatric Cardiology in Sri Lanka has come a long way over a very short period of time. Team work, commitment towards a common goal, and free healthcare are the three main pillars of success. A good system of record keeping, even at its initial stages, was a major factor that has helped us to prove our work and successes in a scientific way. What we have achieved up to now should not be the end of the story as we have come only half way in our journey. We should plan the future based on the lessons learnt from the past, even dating back to the days of Werner Fossman and Robert Gross, and avoid situations which will hinder steady progress. We should make use of advancing technology not only in the field of medicine, but also in other fields like information technology, telecommunication and transport, to provide a quality, cost-effective and sustainable service. We should have the courage to change the existing practices and laws to provide a better quality service. Our target should be to provide a cost-effective service for the majority of patients. Attempting to serve $100 \%$ will make the system a failure as attempting to treat the most complex CHD is not cost-effective in a developing country. We should be open minded and have the courage to change the existing system for sustainable better care. We should be able to forecast the possible shortcomings that could come up with such changes but should not make them a reason to reject the change if the overall benefit to society is more than with the existing system. If we could follow these principles, keep our unity and work towards the common goal of "Patient First", the day that every child with a heart disease gets timely and appropriate treatment will not be that far away.

\section{References}

1. Brady C. The Boeing 737 Technical Site. 1999 Available from: http://www.b737.org.uk/production.htm.

2. Kapur, M. In pursuit of the $\$ 800$ heart surgery 2011 Available from: http:/edition.cnn.com/2011/WORLD/asiapcf/0 4/06/india.health.cities/.

3. Sandhu SK. Transcatheter closure of the atrial septal defect in the elderly. Journal of Invasive Cardiology 2007; 19(12): 513-4.
4. Alexi-Meskishvili VV, Bottcher W. The first closure of the persistent ductus arteriosus. Annals of Thoracic Surgery 90(1): 349-56. http://dx.doi.org/10.1016/j.athoracsur.2010.04. 036

5. Noonan JA. A history of paediatric specialties: the development of paediatric cardiology. Pediatric Research 2004; 56(2): 298-306. http://dx.doi.org/10.1203/01.PDR.0000132662 .73362 .96

6. Casanova $\mathrm{R}$, et al. Cuba's national paediatric cardiology program. MEDICC Review 2010; 12(2): 6-9.

7. Wickramasinghe $P$, Lamabadusuriya SP, Narenthiran S. Prospective study of congenital heart disease in children. Ceylon Medical Journal 2001; 46(3): 96-8.

8. Hoffman JI, Kaplan S. The incidence of congenital heart disease. Journal of the American College of Cardiology 2002; 39(12): 1890-900.

http://dx.doi.org/10.1016/S07351097(02)0188 6-7

9. Macmahon B, McKeown T, Record RG. The incidence and life expectation of children with congenital heart disease. British Heart Journal 1953; 15(2): 121-9. http://dx.doi.org/10.1136/hrt.15.2.121

10. Thanopoulos BD, et al. Closure of atrial septal defects with the Amplatzer occlusion device: preliminary results. Journal of the American College of Cardiology 1998; 31(5): 1110-6. http://dx.doi.org/10.1016/S07351097(98)0003 $9-4$

11. Du ZD, et al. Comparison between transcatheter and surgical closure of secundum atrial septal defect in children and adults: results of a multicenter nonrandomized trial. Journal of the American College of Cardiology 2002; 39(11): 1836-44. http://dx.doi.org/10.1016/S07351097(02)0186 $2-4$

12. Levi DS, Moore JW. Embolization and retrieval of the Amplatzer septal occluder. Catheterization and Cardiovascular Interventions 2004; 61(4): 543-7. http://dx.doi.org/10.1002/ccd.20011 
13. Wilson NJ, et al. Transcatheter closure of secundum atrial septal defects with the Amplatzer septal occluder in adults and children-follow-up closure rates, degree of mitral regurgitation and evolution of arrhythmias. Heart, Lung and Circulation 2008; 17(4): 318-24.

http://dx.doi.org/10.1016/j.hlc.2007.10.013

14. Yilmazer MM, et al. Complications of cardiac catheterization in paediatric patients: a single centre experience. Turkish Journal of Pediatrics 2012; 54(5): 478-85.

15. Mehta $\mathrm{R}$, et al. Complications of paediatric cardiac catheterization: a review in the current era. Catheterization and Cardiovascular Interventions 2008; 72(2): 278-85.

http://dx.doi.org/10.1002/ccd.21580

16. Vitiello R, et al. Complications associated with paediatric cardiac catheterization. Journal of the American College of Cardiology 1998; 32(5): 1433-40.

http://dx.doi.org/10.1016/S07351097(98)0039 6-9

17. Kumar RK. Teamwork in paediatric heart care. Annals of Pediatric Cardiology 2009; 2(2): 140-5.

http://dx.doi.org/10.4103/0974-2069.58315

18. Stark JF, et al. Assessment of mortality rates for congenital heart defects and surgeons' performance. Annals of Thoracic Surgery 2001; 72(1): 169-75.

http://dx.doi.org/10.1016/S00034975(01)0268 9-3
19. Welke KF, et al. The complex relationship between paediatric cardiac surgical case volumes and mortality rates in a national clinical database. Journal of Thoracic and Cardiovascular Surgery 2009; 137(5): 113340.

http://dx.doi.org/10.1016/j.jtcvs.2008.12.012

20. Leblanc JG. Creating a global climate for paediatric cardiac care. World Journal of Pediatrics 2009; 5(2): 89-92.

http://dx.doi.org/10.1007/s12519-009-0019-0

21. McCabe C, Claxton K, Culyer AJ. The NICE cost-effectiveness threshold: what it is and what that means. Pharmacoeconomics 2008; 26(9): 733-44.

http://dx.doi.org/10.2165/00019053200826090 $-00004$

22. Edejer T-T, Baltussen TR, Adam T, Hutubessy R, Acharya A, Evans DB, et al. Making choices in health: WHO guide to costeffectiveness analysis. Geneva: World Health Organization; 2003.

23. Gottret P, Schieber GJ, Waters HR, editors. Good practices in health financing: lessons from reforms in low and middle-income countries. Washington: World Bank; 2008. http://dx.doi.org/10.1596/978-0-8213-7511-2

24. Rannan-Eliya RP, Sikurajapathy L. Sri Lanka: "Good Practice" in Expanding Health Care Coverage. Colombo: World Bank; 2009.

25. Safe and Sustainable Review of Children's Congenital Heart Services in England: Report of the public consultation. 2011. p. 115 . 